\title{
Editorial
}

\section{What is new in valvular heart disease ESC Guidelines 2021?}

Since the 2017 guidelines on the management of valvular heart disease (VHD) were released, increasing attention has been focused on the timing and modalities of both percutaneous and surgical intervention (1). Additionally, new scientific evidence has arisen regarding non-invasive evaluation of VHD and antithrombotic therapies in patients with surgical or transcatheter bioprostheses requiring updated recommendations.

Here we summarize the main novelties in new 2021 ESC guidelines on VHD.(2)

\section{Management of atrial fibrillation (AF) in patients with} native VHD

While according to 2017 guidelines surgical excision or external clipping of the left atrial appendage (LAA) might be considered, latest guidelines suggest LAA occlusion should be considered in all patients with CHA2DS2VASc score $\geq 2$ undergoing valve surgery. In AF patients with aortic stenosis, aortic regurgitation (AR) or mitral regurgitation (MR), direct oral anticoagulants (DOACs) are now preferred over vitamin $\mathrm{K}$ antagonists (VKAs) for stroke prevention.

\section{Recommendations on indications for surgery in severe aortic regurgitation}

Left ventricular (LV) end diastolic diameter (LVEDD) is no more considered when evaluating timing for intervention in asymptomatic patients with severe aortic regurgitation (AR). Current guidelines recommend valve surgery in case of left ventricular end systolic diameter (LVESD) > $50 \mathrm{~mm}$ or LVESD > 25 $\mathrm{mm} / \mathrm{m} 2$ or resting LV ejection fraction (EF) $\leq 50 \%$. Furthermore, a new criterion has been added despite weak class of recommendation (COR) and level of evidence (LOE): surgery may be considered in asymptomatic patients with LVESD $>20 \mathrm{~mm} / \mathrm{m} 2$ or resting LVEF $\leq 55 \%$ if surgery is at low risk (IIB C).
Recommendations on indications for surgery in aortic root or tubular ascending aortic aneurysm (irrespective of the severity of aortic regurgitation) In young patients with aortic root dilatation, valvesparing aortic root replacement is now recommended instead of aortic valve repair using the reimplantation or remodelling with aortic annuloplasty technique.

Recommendations on indications for intervention in symptomatic and asymptomatic aortic stenosis

In patients with severe symptomatic high-grade aortic stenosis, intervention is recommended in case of coexistence of mean gradient $\geq 40 \mathrm{mmHg}$, peak velocity $\geq 4 \mathrm{~m} / \mathrm{s}$ and valve area $\leq 1 \mathrm{~cm}^{2}$ (or $\leq 0.6 \mathrm{~cm}^{2} / \mathrm{m}^{2}$ ) at echocardiographic assessment. According to 2017 guidelines, mean gradient $\geq 40 \mathrm{mmHg}$ or peak velocity $\geq 4 \mathrm{~m} / \mathrm{s}$ were enough to define severe high-gradient aortic stenosis. The criterion for intervention in patients with asymptomatic severe aortic stenosis and LVEF $>55 \%$ has been revised. Severe pulmonary hypertension ( $>60 \mathrm{mmHg}$ confirmed by invasive measurement) with no other explanation is no longer considered one of the parameters to be considered for intervention evaluation. In addition, if severe valve calcification with $V$ max progression $\geq 0.3 \mathrm{~m} / \mathrm{s} /$ year and markedly elevated B-type natriuretic peptide levels ( $>3 \times$ age- and sexcorrected normal range) confirmed by repeated measurements and without other explanation have been reproposed as criteria aiding in decision making, the definition of very severe aortic stenosis has been redefined: mean gradient $\geq 60 \mathrm{mmHg}$ or $V \max \geq 5 \mathrm{~m} / \mathrm{s}$, instead of $V \max >5.5 \mathrm{~m} / \mathrm{s}$.

In addition, new guidelines recommend considering intervention in asymptomatic patients with severe aortic stenosis and systolic LV dysfunction (LVEF <55\%) without another cause (Ila B).

Address for correspondence: Giuseppe Iuliano, Cardio-Thoracic-Vascular Department, University Hospital "San Giovanni di Dio e Ruggi d'Aragona", Heart Tower - Room 807,Largo Città d'Ippocrate, 84131 Salerno, Italy Phone: +39 3465335232 E-mail: gi.iuliano93@gmail.com

Received: 27.11.2021 Accepted: 27.11.2021 


\section{Recommended mode of intervention in patients with aortic stenosis}

The choice between surgical aortic valve replacement (SAVR) and transcatheter aortic valve implantation (TAVI) represents one of the most discussed issue of recent years due to increasing evidence showing noninferiority or even superiority of TAVI compared with SAVR, no more exclusively in high-risk setting (3-6). The role of the Heart Team in aiding this choice is largely enhanced in new guidelines. The choice between surgical and transcatheter intervention must be based upon careful evaluation of clinical, anatomical and procedural factors by the Heart Team, tailoring the right strategy to the right patient after balancing risks and benefits of each approach.

New guidelines recommend SAVR in patients $<75$ years with STS-PROM/EuroSCORE \| $<4 \%$ or in patients unsuitable for transfemoral TAVI and recommend TAVI in patients $\geq 75$ years with STS-PROM/EuroSCORE II $>8 \%$ or unsuitable for surgery, while 2017 guidelines recommend TAVI exclusively in patients not suitable for SAVR after Heart Team evaluation. For all patients not clearly fulfilling criteria recommending SAVR or TAVI, the Heart Team should carefully evaluate the right therapeutic strategy and discuss with the patient who can then make an informed treatment choice.

\section{Indications for intervention in severe primary mitral regurgitation}

Surgery is still recommended in asymptomatic patients with LV dysfunction indicated by LVEF $\leq 60 \%$ and/or LVESD $\geq 40 \mathrm{~mm}$ (instead of $\geq 45 \mathrm{~mm}$ of 2017 guidelines) and should be considered in patients without signs of LV dysfunction with AF secondary to MR or with systolic pulmonary artery pressure (sPAP) at rest $>50 \mathrm{mmHg}$ indicating pulmonary hypertension.

Interestingly, the recent Mitral Regurgitation International Database score has been proposed to estimate the risk of all-cause mortality in patients with severe primary mitral regurgitation due to flail leaflet, treated with guidelines-directed medical therapy (GDMT) or with surgery. Among the variables included in the score, left atrium (LA) diameter $\geq 55 \mathrm{~mm}$ and LVESD $\geq 40 \mathrm{~mm}$ are new thresholds that have been included in the current recommendations. Accordingly, the presence of a flailing mitral leaflet without subsequent structural abnormalities involving LV or LA is no more considered an indication to mitral valve (MV) surgery in low-risk asymptomatic patients with severe PMR. It should be emphasized that when surgery is considered by Heart Team and the results are expected to be durable, $M V$ repair for severe primary $M R$ is the surgical intervention of first choice since it is associated with better survival compared to MV replacement. When repair is not feasible, MV replacement with preservation of the subvalvular apparatus should be performed. Furthermore, an early surgical approach should be considered especially involving a high volume referral center with well-trained surgeons for mitral valve surgery.

Indications for mitral valve intervention in chronic severe secondary mitral regurgitation

As regard to secondary $M R$ (SMR), new guidelines recommend treating, by surgical or percutaneous approach, only patients with severe SMR who remain symptomatic despite GDMT (including cardiac resynchronization therapy if indicated) after careful evaluation in Heart Team. Indications for isolated MV surgery in SMR remains restrictive, particularly due to the absence of proven survival benefit along with significant procedural risk and high rates of recurrent MR. Hence, valve surgery is recommended in patients with severe symptomatic SMR undergoing coronary artery bypass grafting (CABG) or other cardiac surgery. Interestingly, a new recommendation is listed regarding patients with severe SMR and concomitant coronary artery disease or other cardiac disease requiring treatment: in symptomatic patients considered ineligible for surgery by Heart Team, percutaneous coronary intervention ( $\mathrm{PCl}$ ) (and/or TAVI) possibly followed by transcatheter edge-to-edge repair (TEER) should be considered. Contrasting results from two randomized controlled trials published in 2018, MITRAFR and COAPT, have raised more concern about the right selection of the patient candidate for TEER and lead to increasing need for additional studies which allow to better identify patients who will benefit the most from this procedure $(7,8)$. Patients in COAPT trial demonstrated greater severity of SMR and less LV dilatation than those enrolled in MITRA-FR. Perhaps reflecting greater severity of SMR in relation to LV dimensions ('disproportionate' mitral regurgitation), patients in COAPT were overall more likely to benefit from TEER in terms of reduced mortality and heart failure hospitalization. 
Thereby new VHD guidelines state that, among patients without concomitant coronary artery disease or other cardiac disease requiring treatment, TEER should be considered in selected cases when surgery is not suitable and criteria suggesting an increased chance of responding to therapy are fulfilled

On the other hand, when baseline patient characteristics are not suggestive of favorable outcome, TEER may still be a therapeutic option despite lower COR and LOA (IIB C).

\section{Indications for intervention in secondary tricuspid regurgitation}

Reoperation on tricuspid valve after left-sided surgery is a high-risk surgery. Compared to 2017 guidelines, in new guidelines surgery should be considered regardless of previous left-sided surgery in patients with severe secondary tricuspid regurgitation (TR) symptomatic or asymptomatic/mildly symptomatic with right ventricular (RV) dilatation or initial declining in RV function, in absence of severe RV or LV dysfunction or severe pulmonary vascular disease/hypertension. For the first time, 2021 ESC guidelines on VHD listed transcatheter treatment as therapeutic option of symptomatic severe secondary TR, despite being still poorly supported (IIB C).

\section{Recommendations for prosthetic valve selection}

Bioprostheses are no longer recommended only in patients whose life expectancy is lower than the presumed durability of the bioprosthesis, but also when good-quality anticoagulation is unlikely (adherence problems, not readily available) or contraindicated because of high bleeding risk. Additionally, bioprostheses are favored over mechanic prosthesis in patients already on long-term DOACs due to the high risk for thromboembolism (IIb B).

\section{Recommendations for perioperative and postoperative antithrombotic management of valve replacement or repair}

Many novelties have been added concerning management of antithrombotic therapy in the perioperative period. When interruption of oral anticoagulants $(O A C)$ is needed before surgery, bridging therapy is recommended in patients with mechanical prosthetic valve, AF with significant mitral stenosis, AF with CHA2DS2-VASc score $\geq 3$ for women or 2 for men, acute thrombotic event within the previous 4 weeks, and high acute thromboembolic risk. In patients who have undergone valve surgery with indication for postoperative bridging, it is recommended to start unfractionated heparin (UFH) or low molecular weight heparin (LMWH) 12-24 hours after surgery. In patients with mechanical heart valves, it is recommended to (re)-initiate VKAs on the first postoperative day. VKAs must be discontinued before elective surgery to aim for an INR $<1.5$, regardless the need for bridging therapy. To maintain aspirin therapy, whereas indicated, during the periprocedural period is recommended. In patients treated with dual antiplatelet therapy (DAPT) after recent (>30 days) $\mathrm{PCl}$ who need to undergo heart valve surgery, in the absence of an indication for OAC, it is recommended to resume the $\mathrm{P} 2 \mathrm{Y} 12$ inhibitor postoperatively, as soon as there is no concern over bleeding. Moreover, in the same specific setting, bridging P2Y12 with glycoprotein IIb/IIla inhibitors or cangrelor may be considered.

The section related to patients with an indication to concomitant antiplatelet therapy has been revised according to latest evidence in terms of duration of dual and triple antithrombotic therapy in patients with previous $\mathrm{PCl}$ for acute or chronic coronary syndrome (911).

Indications about antithrombotic therapy after surgical valve replacement have been widely revised. After 3 months of oral anticoagulation using VKAs, DOACs should be considered for long-term anticoagulation in patients with $\mathrm{AF}$ who underwent surgical implantation of a bioprosthetic heart valve. In patients with $A F$ undergoing surgical replacement of $M V$ with a bioprosthetic heart valve, however, DOACs may be considered even within 3 months after surgery. While using low-dose aspirin (75-100 mg/day) over oral anticoagulation was encouraged (Ila C vs IIb C) in 2017 guidelines for 3 months following surgical implantation of an aortic bioprosthesis, according to new guidelines low-dose aspirin or oral anticoagulation using VKAs are equally recommended (Ila B). In patients undergoing TAVI, single antiplatelet therapy (SAPT) has been shown to be safer than DAPT. Accordingly, new guidelines strongly recommend lifelong SAPT after TAVI (I A) instead of 3-6 months of DAPT following by lifelong SAPT (Ila C), as suggested by 2017 guidelines. 


\section{Recommendations on management of prosthetic valve dysfunction}

Thrombus on bioprosthetic heart valves may present not only has as clinical valve thrombosis with elevated gradients, but also with hypo-attenuated leaflet thickening (HALT) with or without reduced leaflet motion. Although clinical significance of HALT is still uncertain, OAC in selected patients with confirmed HALT (by transthoracic or transesophageal echocardiography, cinefluoroscopy or cardiac computed tomography) should be considered, at least until resolution.

Regarding bioprosthetic failure, reoperation is still recommended in symptomatic (IC) or asymptomatic (IlaC) patients with significant increase of transprosthetic gradient or severe regurgitation. As the safety and efficacy of transcatheter valve-in-valve implantation in aortic position has been already established, in new guidelines recommendations regarding transcatheter valve-in-valve implantation in the mitral and tricuspid position (IIb $B$ ) in selected patients at high risk for reoperation take place for the first time.

Giuseppe Iuliano, Rodolfo Citro Cardio-Thoracic-Vascular Department, University Hospital "San Giovanni di Dio e Ruggi d'Aragona", Largo Città d'Ippocrate, Salerno, Italy. Peer-review: Internal Conflict of interest: None to declare Authorship: G.I. and R.C are equally contributed to preparation of manuscript and fulfilled authorship criteria Acknowledgement and funding: None to declare

\section{References}

1.Baumgartner H, Falk V, Bax JJ, De Bonis M, Hamm C, Holm PJ, et al. 2017 ESC/EACTS Guidelines for the management of valvular heart disease. Eur Heart J 2017; 38: 2739-91.

2.Vahanian A, Beyersdorf F, Praz F, Milojevic M, Baldus S, Bauersachs J, et al. 2021 ESC/EACTS Guidelines for the management of valvular heart disease. Eur Heart J 2021.

3.Leon MB, Smith CR, Mack MJ, Makkar RR, Svensson LG, Kodali SK, et al. Transcatheter or surgical aortic- valve replacement in intermediate-risk patients. N Engl J Med 2016; 374: 1609-20.

4.Reardon MJ, Van Mieghem NM, Popma JJ, Kleiman NS, Søndergaard L, Mumtaz $M$, et al. Surgical or transcatheter aortic-valve replacement in intermediaterisk patients. N Engl J Med 2017; 376: 1321-31.

5.Mack MJ, Leon MB, Thourani VH, Makkar R, Kodali SK, Russo $\mathrm{M}$, et al. Transcatheter aortic-valve replacement with a balloon-expandable valve in low-risk patients. $\mathrm{N}$ Engl J Med 2019; 380: 1695-705.

6.Popma JJ, Deeb GM, Yakubov SJ, Mumtaz M, Gada H, O'Hair D, et al. Transcatheter aortic-valve replacement with a self-expanding valve in low-risk patients. N Engl J Med 2019; 380: 1706-15.

7.Obadia JF, Messika-Zeitoun D, Leurent G, lung B, Bonnet $\mathrm{G}$, Piriou $\mathrm{N}$, et al. Percutaneous repair or medical treatment for secondary mitral regurgitation. $\mathrm{N}$ Engl J Med 2018; 379: 2297-306.

8.Stone GW, Lindenfeld J, Abraham WT, Kar S, Lim DS, Mishell JM, et al. Transcatheter mitral-valve repair in patients with heart failure. N Engl J Med 2018; 379: 2307-18.

9.Knuuti J, Wijns W, Saraste A, Capodanno D, Barbato E, Funck-Brentano C, et al. 2019 ESC Guidelines for the diagnosis and management of chronic coronary syndromes. Eur Heart J 2020; 41: 407-77.

10.Hindricks G, Potpara T, Dagres N, Arbelo E, Bax JJ, Blomström-Lundqvist C, et al. 2020 ESC Guidelines for the diagnosis and management of atrial fibrillation developed in collaboration with the European Association for Cardio-Thoracic Surgery (EACTS): The Task Force for the diagnosis and management of atrial fibrillation of the European Society of Cardiology (ESC) Developed with the special contribution of the European Heart Rhythm Association (EHRA) of the ESC. Eur Heart J 2021; 42: 373-498.

11.Collet JP, Thiele $H$, Barbato $E$, Barthélémy $O$, Bauersachs J, Bhatt DL, et al. 2020 ESC Guidelines for the management of acute coronary syndromes in patients presenting without persistent ST-segment elevation. Eur Heart J 2021; 42: 1289-367. 
Heart, Vessels and Transplantation 2021; 5: doi: 10.24969/hvt.2020.291

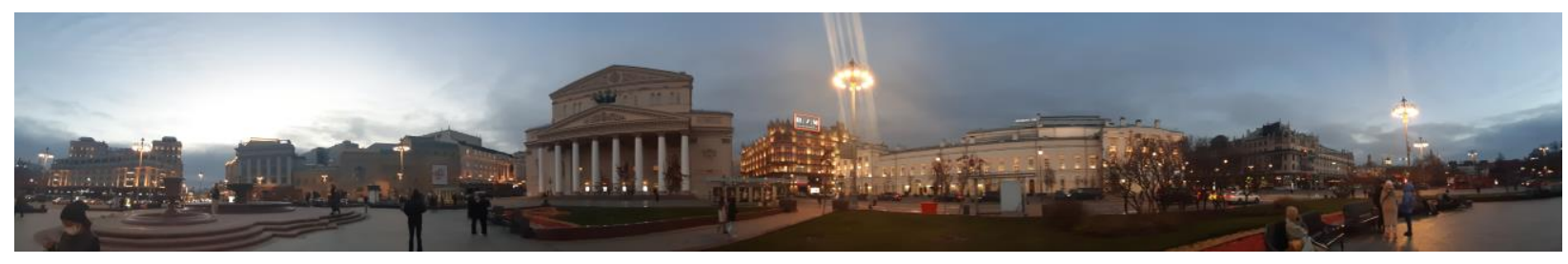

Bolshoi and Malyi Theatres, Teatralnaya square, Moscow, Russia. Shirin Talapbek Kyzy, Bishkek, Kyrgyzstan 